Los Alamos National Laboratory for three years in the area of plasma diagnostics for magnetic fusion energy research. He joined Bell Laboratories in 1982 where he is a member of the Electronic Components and Subsystems Laboratory. He is presently engaged in developing optical devices for wavelength division multiplexing of lightwave systems.

Dr. Lipson is a member of the American Physical Society.

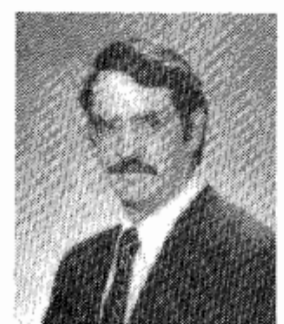

George T. Harvey (M'81) was born in Baltimore, MD in 1949. He received the B.S. degree in electrical engineering from Lehigh University, Bethlehem, PA, in 1974, and the Ph.D. in optics from the University of Rochester, Rochester, NY in 1980.

Since 1980 he has been a Member of the Research Staff at the Engineering Research Center of Western Electric in Princeton, NJ. He is currently working in the area of microoptic devices for optical communication.

Dr. Harvey is a member of the Optical Society of America.

\title{
Broad-Band Ultrasonic Sensor Based on Induced Optical Phase Shifts in Single-Mode Fibers
}

\author{
RAMON P. DE PAULA, MEMBER, IEEE, JAMES H. COLE, AND JOSEPH A. BUCARO
}

\begin{abstract}
A broad-band ultrasonic sensor based on induced optical phase shifts in single-mode fibers is demonstrated over a frequency regime of $0.5-50 \mathrm{MHz}$. In addition, a recently developed theory used to predict the magnitudes of acoustically induced strains in optical fibers is verified.
\end{abstract}

\section{INTRODUCTION}

$\mathrm{R}^{\mathrm{r}}$ ECENTLY, theoretical analysis which predicts the acoustically induced phase shifts and birefringence response for single-mode fibers excited with an ultrasonic wave $(>0.1$ $\mathrm{MHz}$ ) has been published by Flax et al. [1] . The applicability of the model for predicting the birefringence response has recently been demonstrated by the measurements of De Paula et al. [2], [3]. However, from the birefringent case, the values of the optical phase shifts cannot be uniquely determined, since the induced birefringence $\Delta \beta$ is the difference between the orthogonal phase shifts associated with each principal axis, i.e., $\Delta \beta=\Delta \beta_{\|}-\Delta \beta_{\perp}$, where $\Delta \beta_{\|, \perp}$ are the induced phase shift for the parallel and perpendicular polarizations of the $\mathrm{HE}_{11}$ mode, respectively. Here we independently measure in

Manuscript received January 18, 1983; revised March 9, 1983.

The authors are with the Naval Research Laboratory, Washington, DC 20375. the ultrasonic frequency regime each orthogonal phase shift $\Delta \beta_{\|, \perp}$ associated with each principal axis and demonstrate experimentally a very broad frequency response for fiber-optic interferometric sensors.

\section{BACKGROUND}

Considerable effort has been expended toward developing a comprehensive understanding of the pressure-induced phase shifts in single-mode fibers: from the axially unconstrained regime [4], characterized by $l \ll \Lambda$, where $l$ is the length of the fiber and $\Lambda$ is the acoustic wavelength; through the axially constrained regime [5], where $d \ll \Lambda$, but $l>\Lambda$ where $d$ is the fiber diameter; to the anisotropic regime [1], where $d \geqslant \Lambda$. The optical phase shift due to acoustically induced strains can be written, for all of these cases, from the concept of index ellipsoid for the polarized eigenmodes as [6]

$$
\begin{aligned}
& \Delta \beta_{\|}=k_{0} n_{0} l\left[e_{3}-\frac{n_{0}^{2}}{2}\left(P_{11} e_{1}+P_{12} e_{2}+P_{12} e_{3}\right)\right] \\
& \Delta \beta_{\perp}=k_{0} n_{0} l\left[e_{3}-\frac{n_{0}^{2}}{2}\left(P_{12} e_{1}+P_{11} e_{2}+P_{12} e_{3}\right)\right]
\end{aligned}
$$

where $e_{1}, e_{2}$, and $e_{3}$ represent the principal strains of the orthogonal axis, $k_{0}$ is the free-space optical wavenumber, $n_{0}$ is 
the refractive index of the core, $l$ is the acoustooptic interaction length, and $P_{11}$ and $P_{12}$ are the elastooptic coefficients.

An applied acoustic pressure at low frequencies, $(\Lambda \gg l)$ induces an axial strain, $e_{3}=e_{z}$, corresponding to a change in the optical-fiber length and a uniform radial strain $e_{r}=e_{1}=e_{2}$. For this axially unconstrained case, the optical phase shift $\Delta \beta_{u}=\Delta \beta_{\|}=\Delta \beta_{\perp}$ due to the induced strains can be written from (1a) or (1b) as

$$
\Delta \beta_{u}=k_{0} n_{0} l\left[e_{3}-\frac{n_{0}^{2}}{2}\left[\left(P_{11}+P_{12}\right) e_{r}+P_{12} e_{3}\right]\right] \text {. }
$$

As the frequency increases, a transition region between nearsonic and ultrasonic occurs, where the behavior changes from axially unconstrained to axially constrained [4]. Beyond this transition region, the fiber behaves axially constrained $\left(e_{3}=0\right)$ and in this frequency region, the ultrasonic pressure applied to the fiber is still axysymmetric and uniform, i,e., $e_{r}=e_{1}=e_{2}$. Thus $\Delta \beta_{C}=\Delta \beta_{\|}=\Delta \beta_{\perp}$ yielding a single phase shift from either $(1 \mathrm{a})$ or $(1 \mathrm{~b})$

$$
\Delta \beta_{C}=-k_{0} \ln _{0}^{3}\left(P_{11}+P_{12}\right) e_{r} / 2 .
$$

Finally, for a region where $20 d \geqslant \Lambda \geqslant d / 4$, the single-mode fiber is again axially constrained $\left(e_{3}=0\right)$, but the induced principal strains $e_{1}$ and $e_{2}$ are no longer uniform. The optical phase shifts are obtained from (1a) and (1b) upon appropriate calculation and insertion of $e_{1}$ and $e_{2}$. The principal strains $e_{1}$ and $e_{2}$, induced in a single-mode optical fiber of radius " $a$ " by a ultrasonic wave propagating in a fluid, is calculated by solving the displacement wave equation [1], [2], [7]

$$
\boldsymbol{U}=\boldsymbol{\nabla} \phi+\nabla \times \boldsymbol{\psi}
$$

where $\phi$ is the scalar potential and $\boldsymbol{\psi}$ is the vector potential.

If we assume that the plane wave is infinite in extent and that the cylinder is infinitely long, there will be no dependence on the coordinate $z$. The solutions of the scalar and vector potentials are

$$
\phi=\sum_{n} A_{n} J_{n}\left(k_{L} r\right) \cos (n \theta) \exp \left[i \omega_{0} t\right]
$$

and

$$
\psi_{z}=\sum_{n} B_{n} J_{n}\left(k_{s} r\right) \sin (n \theta) \exp \left[i \omega_{0} t\right]
$$

The density, compressional, and shear wavenumbers in the solid material are $\rho_{s}, k_{L}$, and $k_{s}$, respectively. The acoustic wavenumber in the surrounding fluid (assumed to have a density $\left.\rho_{w}\right)$ is $k$ and $\omega_{0}$ is the frequency of the ultrasonic wave. In order to evaluate the unknown coefficients $A_{n}$ and $B_{n}$, it is necessary to apply three boundary conditions that hold at the surface of the fiber, i.e., $r=a$. The displacement and normal stress must be continuous and the tangential stress must be zero at the glass medium boundary. The displacements and strains are then obtained in cylindrical coordinate by the "displacement-potential" and "strain-displacement" relations, respectively, as shown in the analysis of Flax et al. [1].

Diagonalization of the strain matrix yields the induced principal strains as follows

$$
e_{1}=\frac{e_{r r}+e_{\theta \theta}}{2}+\frac{1}{2}\left[\left(e_{r r}-e_{\theta \theta}\right)^{2}+4 e_{r \theta}^{2}\right]^{1 / 2}
$$

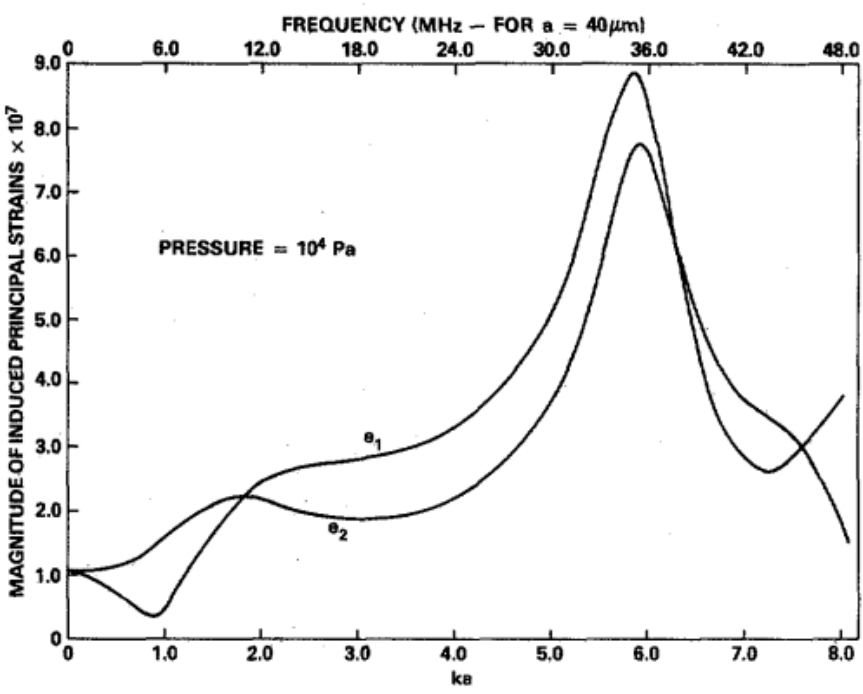

(a)

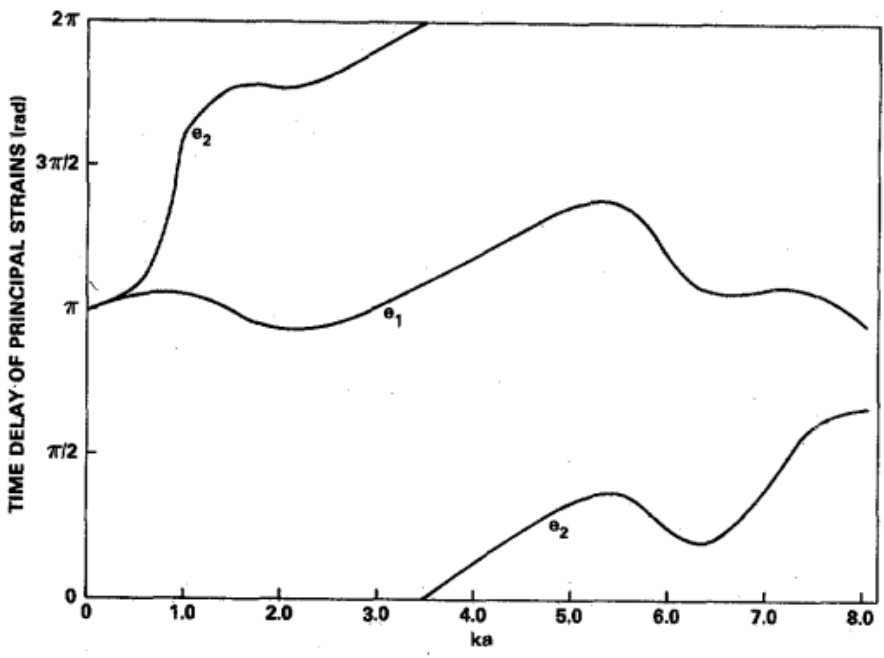

(b)

Fig. 1. (a) The absolute magnitude of the principal strains, $e_{1}$ and $e_{2}$, calculated as a function of $k a$, where $k$ is the acoustic wavenumber and $a$ is the fiber radius. (b) The relative time delay of the principal strains at the fiber core with respect to the acoustic signal incident on the fiber.

and

$$
e_{2}=\frac{e_{r r}+e_{\theta \theta}}{2}-\frac{1}{2}\left[\left(e_{r r}-e_{\theta \theta}\right)^{2}+4 e_{r \theta}^{2}\right]^{1 / 2}
$$

where $e_{r r}, e_{\theta \theta}$, and $e_{r \theta}$ are the strains in cylindrical coordinates. The optical phase shifts are given from (1a) and (1b) as

$$
\begin{aligned}
& \Delta \beta_{\|}=-k_{0} n_{0}^{3} l\left(P_{11} e_{1}+P_{12} e_{2}\right) / 2 \\
& \Delta \beta_{\perp}=-k_{0} n_{0}^{3} l\left(P_{12} e_{1}+P_{11} e_{2}\right) / 2 .
\end{aligned}
$$

Fig. 1 illustrates the magnitude and the relative time delay of the principal strain components as a function of " $k a$," where " $k a$ " is a nondimensional frequency constant, where $k=2 \pi / \Lambda$, $\Lambda$ is the acoustic wavelength, and " $a$ " is the radius of the fiber.

It should be noted that in the original work of the paper by De Paula et al. [2], the relative time delay of the principal strains was, not properly taken into account in computing the magnitude of the optical phase shift. 


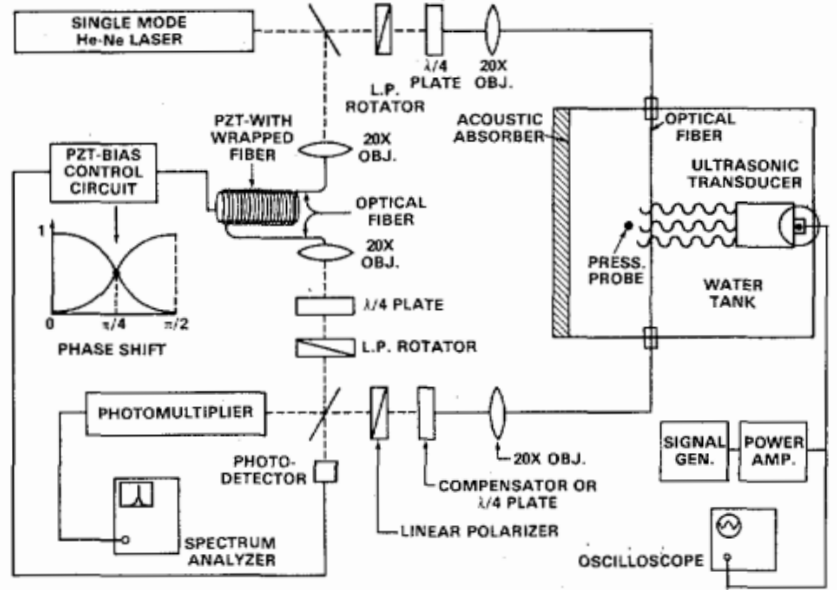

Fig. 2. Experimental set up for interferometric measurement of the two orthogonal optical phase shifts.

\section{EXPERIMENTAL RESULtS}

Measurements of ultrasonically induced phase shifts were made on ITT single-mode fịbers with $80-\mu \mathrm{m}$ o.d. and $4.0-\mu \mathrm{m}$ core for comparison with the calculated phase shifts referred to above. The schematics of the homodyne fiber-optic interferometer used to measure the induced phase shift in each of the orthogonal polarizations is shown in Fig. 2. It is basically a Mach-Zehnder interferometer assembled in the following manner: an optical beam from a single-frequency He-Ne laser with a fixed plane of polarization enters a beam splitter, the splitter forming the reference and sensing beam. The sensing beam passes through a linear polarization rotator emerging at any desired polarizing angle. The light enters a quarter-wave plate and the sensing beam is then focused through a $20 \mathrm{X}$ microscope objective into the sensing fiber. A section of the sensing fiber is submerged into a water tank and a small length interacts with the ultrasonic wave. Another microscope objective at the exit end of the fiber collimates the light output. The output optical beam passes through a quarterwave plate of Soleil-Babinet compensator and then through a Glan-Thompson prism. The reference beam is focused through a $20 \mathrm{X}$ microscope objective into the reference fiber. The reference fiber is wrapped on a PZT cylinder. The PZT is modulated electronically to yield maximum sensitivity to the interferometer, by introducing a static phase shift of $\pi / 2$ between the reference and sensing beam (phase-quadrature condition) [8]. Another $20 \times$ microscope objective at exit end of this fiber collimates the light output. The reference beam passes through a quarter-wave plate and a polarization rotator. The two beams are then recombined on a beam splitter forming two output beams. One proceeds to a photomultiplier and the resulting interference is measured with a spectrum analyzer. The other beam proceeds to a photodetector and produces the error signal [8] necessary to hold the interferometer at quadrature. The error signal is amplified and filtered in a manner similar to that of Jackson et al. [8]. The output of the feedback circuit is applied to the PZT cylinder as the correction signal.

In this experiment, the two polarizations of the $\mathrm{HE}_{11}$ mode are considered separately. Since the goal is to independently measure the induced phase shift in each polarization, only

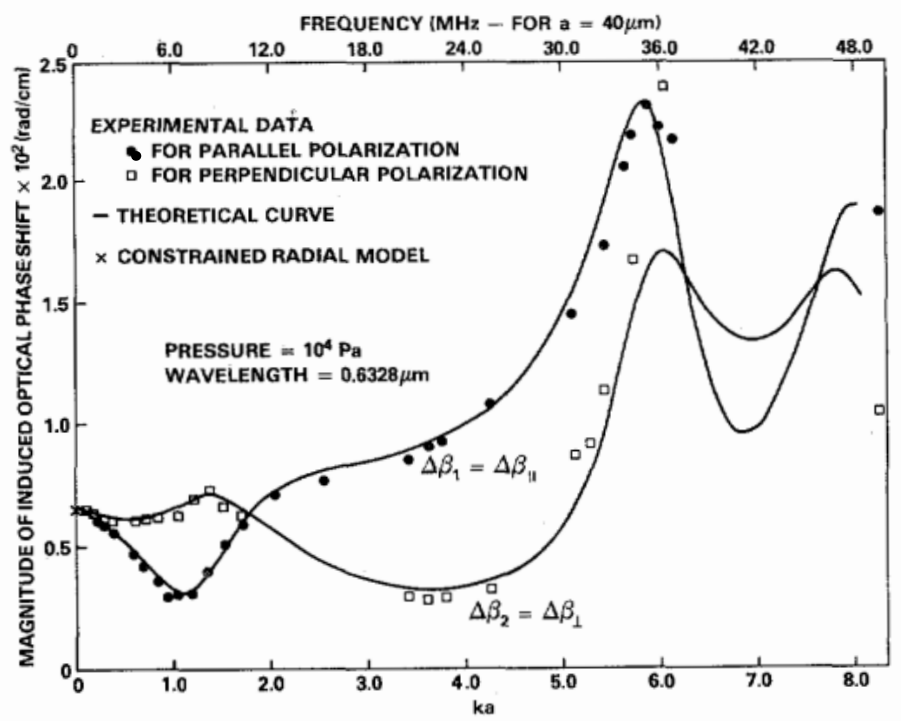

(a)

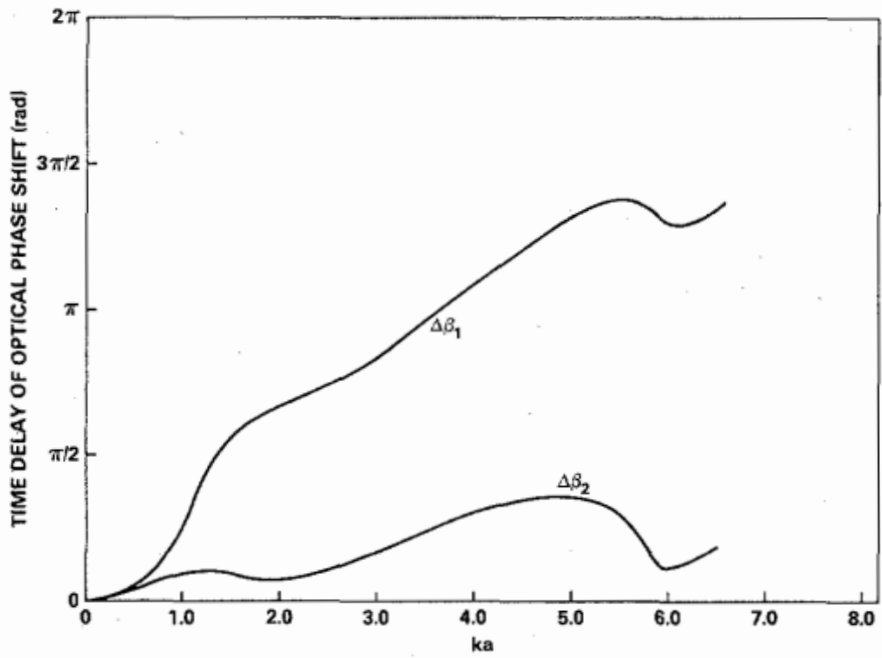

(b)

Fig. 3. (a) The absolute magnitude of induced phase shifts, $\Delta \hat{\beta}_{1}$ and $\Delta \beta_{2}$, calculated and measured as a function of $k a$. (b) The relative time delay of the optical signal from the optical fiber with respect to the acoustic signal incident on the fiber. The relative phase shift is given with respect to a compressive (negative) pressure.

one optical eigenmode must be excited at a time. This is accomplished by aligning the input polarizer and quarterwave plate such that plane polarized light perpendicular or parallel to the induced principal strains arrives at the beginning of the region of interaction. The compensator is adjusted such that the output beam is plane polarized.

With the optical system biased for maximum sensitivity (phase quadrature condition) the output intensity $I$ is [3]

$$
I=I_{0}\left[1+\eta 2 J_{1}\left(\Delta \beta_{i}\right) \sin \omega_{0} t\right] .
$$

$I_{0}$ is the total intensity on the photodetector resulting from the combination of the refererice and the sensing fiber; $J_{1}$ is the first-order Bessel function, $\omega_{0}$ is the frequency of the ultrasonic wave, $\eta$ is the homodyne efficiency, and $\Delta \beta_{i}$ is either $\Delta \beta_{\|}$or $\Delta \beta_{\perp}$.

The magnitude of the measured phase shift is determined from (8). The results are compared with theoretical calculations in Fig. 3 and the agreement is excellent. It is interesting 
to note that the magnitudes of the induced phase shifts are equal at $k a=0$ and $k a \cong 1.8$. Since the induced birefringence is equal to the difference $\Delta \beta_{\|}-\Delta \beta_{\perp}$ the inference that the birefringence is also zero at both these points might be made. However, measurements of this birefringence [2] have shown it to be zero only at $k a \sim 0$ and not at $k a \sim 1.8$. This apparent discrepancy can be understood in the following manner. At $k a=0$ the principal strains are themselves equal, $e_{1}=e_{2}$ (see Fig. 1). However, unlike the case of $k a=0$ where the induced optical phase shifts are equal in magnitude and are in phase with the acoustic signal at the fiber surface, for $k a>0.5$, the phase delay of the optical phase shifts with respect to the incident acoustic field is a complicated function as shown in Fig. 3(b). This phenomenon can be readily understood since, unlike the case where the fiber strains are uniform, the principal strains $e_{1}$ and $e_{2}$ are independent functions of $k a$ (see Fig. 1). It is the fact that the principal strains are out of phase with the incident acoustic field and with each other (see Fig. $3(\mathrm{~b}))$ which leads to the relative phase differences between the optical phase shifts and a resulting birefringence even for equal phase shift magnitudes at $k a \simeq 1.8$. The peak value of the induced optical phase shift occurs at $k a \approx 6(\sim 35.8 \mathrm{MHz}$ for a fiber with $80-\mu \mathrm{m} \mathrm{o.d}$.) and this is due to the optical-fiber half-wavelength resonance $(\Lambda=d / 2)$.

\section{Concluding Remarks}

As is evident from Fig. 3, the phase shifts reported here have been measured over a wide range of frequencies. The response over the entire frequency range is large and varies no more than one order of magnitude. Since the state of the art of packaging optical interferometers for sensing applications is well developed [9], this work demonstrates the potential for a broad-band ultrasonic sensor covering a wide frequency range (0.1-50 MHz). In addition, the magnitude of ultrasonically induced phase shifts in optical fibers have been uniquely determined experimentally and are in excellent agreement with the theoretical model of Flax et al. [1] .

\section{ACKNOWLEDGMENT}

The authors would like to thank A. Dandridge and A. B. Tveten for helpful suggestions concerning the demodulation system.

\section{REFERENCES}

[1] L. Flax, J. H. Cole, R. P. De Paula, and J. A. Bucaro, "Acoustically induced birefringence in optical fibers," J. Opt. Soc. Amer., vol. 72, pp. 1159-1162, Sept. 1982.

[2] R. P. De Paula, L. Flax, J. H. Cole, and J. A. Bucaro, "Single-mode fiber ultrasonic sensor," IEEE J. Quantum Electron., vol. QE-18, pp. 680-683, Apr. 1982.

[3] R. P. De Paula, "Interaction of high frequency acoustic waves and optical waves propagating in single mode fiber," $\mathrm{Ph} . \mathrm{D}$. dissertation, Catholic Univ. of Amer., Wash., DC, 1982.

[4] R. Hughes and J. Jarzynski, "Static pressure sensitivity amplification in interferometric fiber optic hydrophones," Appl. Opt., vol. 19, pp. 98-107, Jan. 1980.

[5] J. Jarzynski, R. Hughes, T. R. Hickman, J. A. Bucaro, "Frequency response of interferometric fiber optic coil hydrophones," $J$. Acoust. Soc. Amer., vol. 69, pp. 1799-1808, 1981.

[6] J. F. Nye, "Physical properties of crystals," London, Oxford, 1979, pp. 243-254.

[7] E. K. Sittig and G. A. Coquin, "Visualization of plane strain vibration modes of a long cylinder capable of producing sound radiation," J. Acoust. Soc. Amer., vol. 48, pp. 1150-1159, Sept. 1970.

[8] D. A. Jackson, R. Priest, A. Dandridge, and A. B. Tveten, "Elimination of drift in a single mode optical fiber interferometer using a piezoelectrically stretched coiled fiber," Appl. Opt., vol. 19, pp. 2926-2929, 1980.

[9] T. G. Giallorenzi et al., "Optical fiber sensor technology," IEEE J. Quantum Electron., vol. QE-18, pp. 626-665, Apr. 1982.

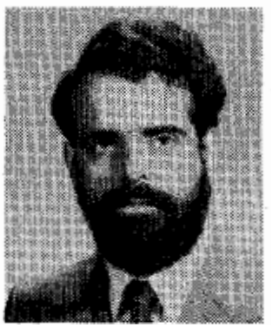

Ramon P. DePaula (M'81) was born in Sao Paulo, Brazil, on November 11,1952 . He received the B.S.E.E. degree from Catholic University of America, Washington, DC, in 1974, and the M.S. degree in nuclear engineering from the University of California, Berkeley in 1979 , and will receive the Ph.D. degree from Catholic University of America in 1982.

From 1974 to 1976 he was a graduate student at the University of California where he worked as a Research Assistant in the Lawrence Berkeley Laboratory on laser-induced vaporization of uranium dioxide. In 1977 he joined the Vitreous State Laboratory, Catholic University, as a graduate student, where he made measurements of properties of glass materials and also worked on characterization of multimode fibers. Since 1979 he has worked at the Physical Acoustics Branch, Naval Research Laboratory, Washington, DC, studying the interaction of ultrasonic waves with single-mode fibers for the development of ultrasonic sensors in fluids, air, and solids. His present research interest includes fiber-optic sensor systems and phase and frequency modulators for single-mode fibers.

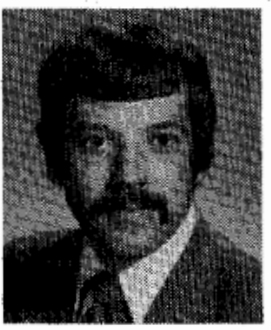

James H. Cole completed graduate work at Pennsylvania State University, University Park, $\mathrm{PA}$, in 1973.

He joined the TRW Defense and Space Systems Group in 1973. Initially, his efforts were aimed at lowering the applicable frequency range of Bragg and Raman Nath imaging systems. An investigation of optical techniques for detection of low-frequency sound followed the imaging work. This effort was extended to fiber-optic detection techniques in 1975, and led to one of the first fiber-optic acoustic sensor publications. Since 1978, he has been with the Naval Research Laboratory, Washington, DC, where he directs and performs research investigating fiber sensor transduction mechanisms. He has numerous publications in the areas of acoustooptic imaging and fiber sensor technology.

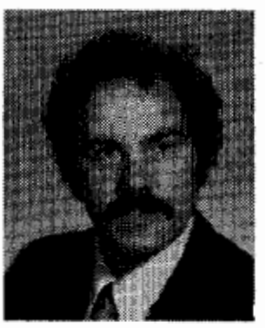

Joseph A. Bucaro received the B.S. and M.S. degrees from John Carroll University, Cleveland, $\mathrm{OH}$, in 1967, and the Ph.D. degree in physics from Catholic University of America, Washington, DC, in 1971.

He joined the Naval Research Laboratory, Washington, DC, in 1971, and carried out research in acoustic surface wave propagation and the mechanical properties of surfaces. In 1973 he formed the Acousto-Optics Section at the NRL and began programs in acoustic imaging, acoustooptic materials, and optical-fiber sensors. He became head of the Physical Acoustics Branch, Naval Research Laboratory, in 1978 where he has been managing a number of programs in acoustics and acoustooptics and is continuing research in optical-fiber optics. 